\title{
Pourquoi les Femmes Arrêtent-Elles D'utiliser la Contraception? L'exemple du Botswana
}

\author{
Pierre NGOM
}

\section{RÉSUMÉ}

Cette étude examine les causes de l'arrêt des méthodes de régulation de la fécondité au Botswana en se basant sur les données de son Enquête Démographique et de Santé menée en 1988. La majorité des femmes qui ont arrêté l'utilisation de leur méthode de planification familiale ont évoqué deux causes principales: les problèmes de santé rencontrés et le désir d'avoir d'autres enfants. L'opposition du mari (ou du partenaire sexuel) est l'une des autres raisons importantes mentionnées par les femmes. L'auteur identifie ensuite les facteurs socioéconomiques et culturels qui peuvent influencer les femmes à arrêter leur méthode de régulation de la fécondité. Finalement, quelques implications pour les programmes africains de planification familiale sont discutées.

\section{INTRODUCTION}

Deux raisons majeures expliquent pourquoi les programmes de planification familiale s'intéressent aux causes et conséquences de l'arrêt de l'utilisation des méthodes contraceptives. D'une part, lorsqu'une femme décide de ne plus pratiquer la contraception, elle est, en moyenne, exposée à un risque beaucoup plus élevé de contracter des grossesses répétées. D'autre part, plusieurs recherches sur l'arrêt des méthodes contraceptives montrent que, le plus souvent, celui-ci reflète la qualité même des services de planification familiale (Bruce 1990, Foreit 1991, Williamson et al. 1991).

Peu de recherches sur l'arrêt de l'utilisation des méthodes contraceptives en Afrique sont actuellement disponibles. Cette situation est certainement due au fait que, dans beaucoup de pays en Afrique au Sud du Sahara, I'un des objectifs principaux des programmes de planification familiale est d'accroître le niveau national de la prévalence contraceptive. Toutefois, une proportion élevée de femmes qui arrêtent l'utilisation de leur méthode de régulation des naissances peut avoir un effet négatif sur la prévalence contraceptive d'un pays donné, même si la proportion de nouvelles recrues qui adoptent la planification familiale reste stable dans le temps. Une autre conséquence de l'arrêt des méthodes contraceptives est que celui-ci peut avoir indirectement un impact négatif sur la proportion de femmes qui décident d'adopter la contraception. Les femmes qui arrêtent d'utiliser la pilule, parce qu'elle leur cause des vertiges par exemple, peuvent en parler à leurs amies qui, conséquemment, seront découragées d'adopter une telle méthode.

Les enquêtes démographiques menées récemment au Botswana montrent que l'indice synthétique de fécondité est passé de 7,1 en 1981 à 6,5 en 1984 et 5,0 en 1988 (Lesetedi et al. 1989). Ces changements sont essentiellement attribuables au succès du programme national de planification familiale mis en place depuis 1973. La proportion des femmes en union, utilisatrices de méthodes modernes de contraception, est passée de $19 \%$ en 1984 à $33 \%$ en 1988 . Toutefois, d'après les données de l'Enquête Démographique et de Santé au Botswana de 1988, plus de la moitié des femmes ayant pratiqué la contraception au cours des cinq dernières années précédant la date de cette enquête ont eu à arrêter l'utilisation d'au moins une méthode.

La présente étude aborde les aspects majeurs de l'arrêt de l'utilisation des méthodes contraceptives au Botswana. Les implications pour les programmes de planification familiale en Afrique y sont également examinées. Plus précisément, nous répondrons aux trois questions suivantes: quelles est l'ampleur de l'arrêt des méthodes de régulation de la fécondité au Botswana? Pourquoi les femmes arrêtent-elles d'utiliser la contraception au Botswana? Et, quelles en sont les implications pour les programmes africains de planification familiale?

Ces questions seront abordées en utilisant les donnés collectées par l'Enquête Démographique 
et de Santé au Botswana (EDSB) de I988. L'EDSB avait interrogé 4.368 femmes âgées de 15 à 49 ans révolus. Pour chaque femme enquêtée, des informations ont été recueillies sur les dates de début d'utilisation et d'arrêt des deux dernières méthodes contraceptives. Pour réduire les biais éventuels dûs aux défauts de mémoire, seule la dernière méthode contraceptive fera l'objet des analyses qui vont suivre. Toutefois, II se peut que certains de ces arrêts soient temporaires: l'enquêtée peut décider d'utiliser une autre méthode immédiatement après la date de l'enquête.

\section{Arrêt des méthodes de régulation de la fécondité au Botswana}

A partir des données de l'EDSB, il est possible de construire une table de survie pour l'arrêt des méthodes de régulation de la fécondité. Une telle table a pour but de présenter le devenir d'une cohorte de contraceptrices durant les 12 premiers mois suivant leur début d'utilisation d'une méthode moderne. Pour la reconstitution de cette cohorte, l'échantillon a été restreinte aux femmes qui ont entamé l'utilisation de leur dernière méthode au cours de la période triannuelle précédant la date de l'enquête. Les probabilités de survie obtenus à chaque durée mensuelle ont été légèrement corrigées pour tenir compte de l'effet de censure introduit par la date de l'enquête. Les résultats d'un tel procédé figurent au Tableau I.

Les résultats du tableau I suggèrent que I7\% des femmes du Botswana qui adoptent une méthode contraceptive l'abandonnent au terme de la première année d'utilisation. En guise de comparaison, le pourcentage correspondant est seulement de $6 \%$ pour des pays comme la Colombie et la Thailande (Bulatao, 1993). Le pourcentage trouvé pour le Botswana est comparable à celui du Paraguay (18\%) qui, selon Bulatao (1993: 43), est considérablement élevé. L'arrêt des méthodes de régulation de la fécondité est donc d'une ampleur sérieuse au Botswana. II est par conséquent important d'identifier les principales causes qui sous-tendent de tels arrêts.

\section{Pourquoi les femmes arrêtent-elles d'utiliser la contraception au Botswana?}

L'enquête Démographique et de Santé du Botswana a demandé à chaque femme, qui a arrêté l'utilisation d'une méthode contraceptive, la raison qui l'a conduite à entreprendre une telle action. La figure I présente les trois principales causes évoquées par les femmes qui ont arrêté une méthode contraceptive dans les trois dernières années précédant la date de l'enquête (les autres causes sont présentées au Tableau 2). Ces causes ont été arrangées selon le type de méthode contraceptive: Pilule, dispositif intrautérin (DIU), et injections. Du fait de la faiblesse des effectifs en présence, les méthodes traditionelles (abstinence, retrait) et le condom ne sont pas inclus au sein du tableau $\mathrm{I}$.

Concernant le DIU, la pilule et les injections, la plupart des femmes ont indiqué qu'elles ont arrêté leur utilisation à cause de problèmes de santé. Bien que I'EDSB n'ait pas collecté des données détaillées sur la nature de ces problèmes de santé, il est bien connu que l'insertion du DIU est souvent associée à des effets secondaires réels tels que des crampes menstruelles ou des saignées anormales (Mauldin 1978). D'autres complications médicales, bien que rares, peuvent être également causées par l'utilisation du DIU: par exemple, inflammations du pelvis, perforations de l'utérus, etc. (Kraeger 1977).

En plus de ces effets secondaires réels, il faut également noter que certains des problèmes de santé évoqués par les femmes peuvent refléter les rumeurs qui circulent à propos des méthodes contraceptives. L'imagerie populaire au Botswana véhicule un ensemble de croyances négatives concernant les méthodes contraceptives et celleslà influencent certainement les femmes qui utilisent de telles méthodes. Larson (1983), au terme d'une enquête sur de telles croyances, montre que la plupart des femmes du Botswana croient que, pendant les relations sexuelles, le DIU peut aller se loger au niveau du cerveau ou de la gorge de la femme; que le DIU cause des maladies vénériennes, et que la taille du DIU augmente énormément au sein de l'organisme de la femme de laquelle elle peut ne plus être enlevée. Cette même étude indique également que les femmes du Botswana croient que la pilule accroît anormalement le poids du nouveau-né et qu'elle cause le cancer. De telles croyances sont effrayantes et peuvent aussi expliquer la 
proportion élevée de femmes qui ont arrêté les injections pour des raisons de santé (Figure I).

La deuxième raison la plus importante évoquée par les femmes qui arrêtent d'utiliser la contraception concerne le désir d'avoir un autre enfant. Ceci reflète certainement, comme l'indiquent van de Walle et van de Walle (I99I), que la demande de contraception pour des besoins d'espacement des naissances reste encore très élevée pour les pays de l'Afrique subsaharienne. Au Botswana, beaucoup de femmes utilisent la contraception pour espacer leurs naissances et arrêtent la pratique de la planification familiale dès qu'elles veulent avoir d'autres enfants. Cependant un certain nombre de femmes qui utilisent la contraception et qui tombent accidentellement enceinte auront tendance à considérer une telle grossesse comme étant désirée.

L'opposition du mari ou du partenaire sexuel est la troisième raison évoquée par les femmes qui ont arrêté l'utilisation de leur méthode de régulation des naissances. La décision d'utiliser la contraception ne dépend pas d'un seul individu: dans les sociétés où les femmes subissent la dictature des hommes (par exemple: Afrique, Japon), le résultat précédant n'est pas surprenant. Que l'influence du mari dans l'arrêt des méthodes contraceptive arrive seulement en troisième position (Figure I) est dû au fait que c'est généralement par la suite d'un consensus au sein du couple que les femmes en question avaient décidé d'utiliser de telles méthodes.

Les raisons qui sous-tendent l'arrêt des méthodes de régulations des naissances sont également liées aux caractéristiques socio-économiques et culturelles des utilisatrices de la contraception.

\section{Influence des variables socio-économiques et culturelles sur l'arrêt de l'utilisation des méthodes contraceptives.}

Quelques exemples suffisent pour introduire ce que nous entendons par variables socioéconomiques et culturelles influençant l'arrêt de l'utilisation des méthodes de régulation des naissances. Par exemple, les femmes n'ayant jamais été à l'école ont probablement plus tendance à arrêter l'utilisation de leur méthode contraceptive pour des raisons de santé étant donné qu'elles sont plus exposées aux rumeurs qui circulent concernant les effets secondaires fictifs de telles méthodes. En outre, les femmes qui ont un niveau scolaire élevé sont généralement plus motivées à utiliser de façon continue la contraception, probablement par ce qu'elles doivent travailler dans le secteur moderne de l'économie ou simplement parce qu'elles sont plus attirées par la famille nucléaire de type occidentale. Les femmes qui n'ont pas beaucoup d'enfants évoqueront certainement leur désir d'avoir d'autres enfants comme étant la raison qui les pousse à arrêter leur méthode de planification familiale. Une bonne connaissance de la nature de ces relations est très importante surtout lorsqu'il s'agit d'élaborer des stratégies de promotion de la planification familiale destinées à des population cibles bien déterminées. Comme l'indiquent Dackam Ngatchou, Mfoulou et Sala-Diakanda (1990: II5): "I'utilisation des services de planning familial dépend évidemment des croyances et des traditions, du niveau d'instruction, etc.... Les APF [Association pour la Planification Familale] doivent en tenir compte dans leurs activités d'IEC pour orienter les information adéquates selon la population cible retenue".

Nous utiliserons un modèle explicatif qui relie le risque mensuel d'arrêter d'utilisation d'une méthode contraceptive et les caractéristiques socio-économiques et culturelles suivantes: le niveau d'éducation de la femme (n'a jamais fréquenté l'école, niveau primaire, niveau secondaire ou plus), son âge à la dernière maternité, le type de méthode contraceptive utilisée (pilule, DIU, injections, autres méthodes), sa religion (chrétienne/protestante, traditionnelle, autre/sans religion) et son milieu de résidence (urbain ou rural). L'influence de ces variables sur l'arrêt des méthodes de régulation des naissances a été démontrée par plusieurs autres études (Grady et al. 1988, Hammerslough 1984, Schirm et al. 1982). Ce modèle permettra de comparer le risque d'arrêter d'une méthode contraceptive pour les groupes de femmes appartenant aux différentes catégories d'une même variable socioéconomique ou culturelle donnée, tout en assurant que les différences qu'elles peuvent avoir sur les autres variables soient éliminées (Tableau $3)$. En d'autres termes, ce modèle permet de répondre à des questions du genre : en supposant que deux femmes utilisent la même méthode 
contraceptive, sont toutes deux du milieu rural, ont le même âge à la dernière maternité, mais ont des niveaux d'éducation différents, quelle est celle qui est soumise à un risque plus élevé d'arrêter d'utiliser la contraception. Les résultats provenant d'un tel modèle sont présentés au tableau 3.

Toutes choses étant égales par ailleurs, le risque d'arrêt de l'utilisation des méthodes de régulation des naissances décroît lorsque le niveau d'éducation augmente. Le tableau 3 indique que les femmes ayant au moins le niveau d'éducation secondaire ont un risque d'arrêt de leur méthode contraceptive $52 \%$ fois moins élevés que celui des femmes sans niveau d'éducation. Aucune différence substantielle n'a été observée entre les femmes de niveau d'éducation primaire et celles n'ayant jamais fréquenté l'école. II est fort plausible qu'en fait le niveau secondaire soit le seuil à partir duquel la motivation des femmes du Botswana pour arrêter la contraception ait un effet significatif. Les femmes de niveau secondaire (ou plus) ont probablement plus de chances de travailler dans le secteur moderne de l'économie, alors que quelques années d'éducation primaire ne permettent nécessairement pas l'accès à de tels débouchés. Mais il est également possible que les femmes ayant au moins le niveau d'éducation secondaire soient plus réceptives aux campagnes d'Information, d'Education et de Communication sur les méthodes de régulation de la fécondité.

Le tableau 3 montre également que le risque d'arrêter la pratique de la régulation des naissances baisse lorsque l'âge à la dernière maternité augmente. Les femmes dont l'âge à la dernière maternité est élevé sont en fait les femmes ayant une parité plus élevée que la moyenne. Ces femmes ont donc certainement plus de chances d'avoir atteint le nombre d'enfants desiré et sont donc beaucoup plus motivées de ne pas arrêter la pratique de la contraception. Les femmes à parité élevée sont également plus motivées à espacer leurs naissances que celles qui n'ont pas beaucoup d'enfants. Grady et Billy (1989) soutiennent que cet effet négatif du nombre d'enfants nés vivants sur le risque de d'arrêter l'utilisation des méthodes de régulation des naissances est lié au fait que les femmes à parité élevée sont plus sensibles aux coûts (monétaires, psychologiques, et sociaux) que leur imposerait une grossesse non planifiée.
Les résultats du Tableau 3 démontrent que les femmes qui utilisent le DIU arrêtent dans une proportion plus faible la pratique de la contraception que celles qui utilisent la pilule. Le rapport des risques - ou risque relatif - d'arrêter l'utilisation de la contraception entre ces deux groupes de femmes est de $38 \%$. Le fait que le DIU soit plus performant que les autres méthodes est partiellement lié à certaines caractéristiques de cette méthode. D'une part la technologie du DIU a été substantiellement modifiée afin de réduire les risques de saignée ou d'inflammation du pelvis (Lee et al. 1988). D'autre part l'efficacité même du DIU a été largement améliorée: Segal(1989) a estimé que moins de $1 \%$ des femmes qui utilisent correctement le TCU-380 contractent une grossesse non désirée. Les autres facteurs qui expliquent la performance du DIU sont relatifs aux caratéristiques des femmes qui l'utilisent. Une fois que le DIU est inséré, il n'est plus sous l'influence de certains facteurs (tels que l'oubli ou l'utilisation incorrecte) qui jouent un rôle négatif sur la pratique continue des autres méthodes contraceptives telles que la pilule ou le condom. II faut noter aussi que les femmes qui utilisent le DIU sont, en général, celles qui veulent strictement réguler leur fécondité. Au Botswana, une femme n'est autorisée d'utiliser le DIU que lorsqu'elle a déjà atteint le nombre d'enfants désiré (voir Lesetedi et al. 1989: 6).

Le tableau 3 suggère enfin que les femmes affiliées aux religions traditionelles africaines arrêtent dans une proportion plus grande les méthodes de régulation des naissances que celles qui sont adeptes des autres religions (protestantisme, catholicisme, et celle n'ayant aucune religion). Selon Caldwell et Caldwell (1988), ces différences sont dues au fait qu'être affilié à une religion africaine signifie être moins exposé aux idées provenant du monde occidental. Mais une telle explication ne peut être soutenue ici puisque les différences dues au niveau d'éducation (qui mesure certainement mieux le degré d'exposition au monde occidental) ont été éliminées. Nous croyons que les différences observées sont liées au fait que religions traditionelles africaines et croyances populaires vont de paire, et que les croyances populaires au Botswana véhiculent un certain nombre de rumeurs qui peuvent décourager l'utilisation continue des méthodes de régulation des naissances. Les religions traditionelles africaines impliquent également une 
conformité stricte avec les exigences des ancêtres qui considèrent la régulation des naissances comme un péché qui mérite une sévère punition (Molnos 1968).

Les causes qui sous-tendent l'arrêt des méthodes de régulation de la fécondité sont donc diverses. Au Botswana, les femmes arrêtent l'utilisation de telles méthodes pour deux raisons essentielles: les problèmes de santé (réels ou fictifs) causés par ces méthodes, et le désir d'avoir d'autres enfants. Une autre raison avancée par les femmes concerne l'opposition de leur mari. Nous avions également montré qu'être affilié à une religion africaine, avoir une parité peu élevée, avoir un bas niveau d'éducation, et utiliser une méthode contraceptive autre que le DIU sont des facteurs qui influencent les femmes à arrêter leur méthode de régulation de la fécondité.

Quelques implications de l'arrêt des méthodes de régulation de la fécondité pour les programmes africains de planification familiale

Pour mieux comprendre l'importance qui doit être accordée à la recherche sur les causes et conséquences de l'arrêt des méthodes de régulation des naissances, il est important d'étudier la relation qui existe entre la proportion de femmes qui arrêtent la pratique de la contraception et le niveau national de la prévalence contraceptive. La figure 2 a été obtenue à partir d'un modèle de simulation comprenant trois variables: la prévalence contraceptive annuelle, la proportion de femmes qui arrêtent leur méthode contraceptive après moins d'un an d'utilisation, et la proportion de nouvelles recrues par le programme national de planification familiale (Williamson et al. 1991, Jain 1989, Mauldin 1978, Bruce et Jain 199I).

Ce modèle de simulation démontre que les efforts investis par les programmes de planification familiale, afin d'augmenter la prévalence contraceptive, peuvent bénéficier d'un effet d'entraînement immense si la proportion de femmes qui arrêtent la pratique de la contraception baisse. Nous avions indiqué que le taux annuel d'arrêt des méthodes contraceptives est de $17 \%$ au Botswana où la prévalence contraceptive est de $33 \%$. Pour maintenir le taux de prévalence contraceptive à ce niveau, il est nécessaire de recruiter, annnuellement, au moins $6 \%$ des utilisatrices potentielles de la contraception. Supposons que, pour les dix prochaines annés, le taux de recruitement reste constant à $6 \%$, mais que le programme national de planification familiale veuille accroître la prévalence contraceptive de $33 \%$ à $50 \%$. Les résultats du modèle présenté au niveau de la figure 2 indiquent qu'en réduisant le taux annuel d'arrêt des méthodes de régulation de la fécondité de $17 \%$ à $12 \%$, la prévalence contraceptive augmenterait de $33 \%$ à $50 \%$.

La réduction de la proportion de femmes qui arrêtent leur méthode de régulation des naissances veut également dire qu'un nombre de plus en plus élevé d'utilisatrices sont satisfaites de leur méthode de choix. Celles ci peuvent en parler à leurs amies et un effet boule de neige peut s'en suivre. Piotrow et Meyer (199I: 109) indiquent que "les utilisatrices satisfaites sont une des sources de communication les plus crédibles quand il s'agit d'atteindre des clients potentiels et peuvent accroître le taux d'adoption [de la contraception]".

Un autre aspect des résultats présentés sur la figure 2 est la suivante: la prévalence contraceptive peut baisser si le taux annuel d'arrêt des méthodes de régulation de la fécondité augmente, et ce, même si la proportion de nouvelles recrues qui adoptent la planification familiale reste stable dans le temps. Les programmes nationaux de planification familiale doivent donc élaborer des stratégies appropriées pour réduire la proportion de femmes qui arrêtent leur méthode de régulation des naissances.

\section{CONCLUSION}

Une proportion élevée de femmes ont arrêté leur méthode de régulation des naissances pour des raisons de santé. En outre, la proportion de femmes qui arrêtent leur méthode contraceptive est beaucoup plus élevée parmi celles ayant un bas niveau d'éducation et celles affiliées aux religions traditionnelles africaines. Un remède partiel à cette situation consiste à améliorer les campagnes d'IEC. Cette amélioration sera mieux réussie si les responsables des programmes de planification 
familiale sont au courant des rumeurs concernant les méthodes contraceptives. Par exemple, si dans une zone donnée, la rumeur la plus persistante est que "la contraception rend stérile", ou que "un bébé $X$ est né avec un stérilet dans la main", les campagnes d'IEC doivent lutter contre de telles croyances. Mais pour mener à bien cette lutte il faut d'abord que le contenu de ces rumeurs soit connu.

La deuxième raison importante mentionnée par les femmes, qui arrêtent d'utiliser les méthodes de régulation des naissances, est qu'elles désirent avoir d'autres enfants. Un autre résultat qui est rapprochable à celà est que les femmes à parité élevée ont plus tendance à arrêter l'utilisation de telles méthodes. Ces deux aspects suggèrent, si besoin en est, que l'arrêt de la pratique de la régulation des naissances est étroitement lié à la fécondité des utilisatrices des méthodes contraceptives. Là intervient le rôle de la motivation de la femme (ou du couple) à exercer un contrôle aussi bien sur son comportement procréateur que sur le choix du type de méthode à utiliser. II est plus indiqué de fournir des méthodes temporaires (pilules, condoms, méthodes vaginales) aux femmes qui veulent espacer leur naissances. Quant à celles qui ne désirent plus avoir d'enfants les méthodes à long terme (DIU) sont plus indiquées.

La présente étude a aussi insisté sur le fait que beaucoup de femmes arrêtent l'utilisation de leur méthode contraceptive suite à des pressions exercées par leur mari. II est donc urgent que les programmes africains de planification familiale prennent en compte l'opinion des hommes. Un rapport élaboré par la Fédération Internationale pour le Planning Familial (IPPF 1984) suggère qu'une façon d'atteindre un tel objectif consiste à mener des activités d'information afin de gagner le support des maris.

Tableau I : Table de survie pour l'arrêt des méthodes de régulation de la fécondité

\begin{tabular}{||l||l||}
\hline \hline Durée d'utilisation & $\begin{array}{l}\text { Proportions } \\
\text { d'utilisatrices de } \\
\text { la contraception }\end{array}$ \\
\hline \hline 0 & 1,0000 \\
1 & 0,9967 \\
2 & 0,9769 \\
3 & 0,9669 \\
4 & 0,9378 \\
5 & 0,9182 \\
6 & 0,9053 \\
7 & 0,8916 \\
8 & 0,8843 \\
9 & 0,8686 \\
10 & 0,8504 \\
\hline 11 & 0,8489 \\
\hline
\end{tabular}

Source: Base de données de l'Enquête Démographique et de Santé au Botswana de 1988.

Tableau 2 : Distribution (en \%) des femmes qui ont arrêté leur dernière méthode de régulation des naissances durant les trois années précédant l'enquête, selon la cause évoquée 


\begin{tabular}{|c|c|c|c|}
\hline \multirow{2}{*}{$\begin{array}{l}\text { Causes de l'arrêt des méthodes } \\
\text { de régulation de la fécondité }\end{array}$} & \multicolumn{3}{|c|}{ Type de Méthode } \\
\hline & Pilule & DIU & Injections \\
\hline Désir d'avoir d'autres enfants & 13.6 & 25.5 & 8.9 \\
\hline Méthode inefficace & 0.3 & 1.3 & 0.0 \\
\hline Opposition du mari & 5.2 & 3.3 & 2.6 \\
\hline Problèmes de santé & 58.9 & 46.4 & 77.1 \\
\hline Difficile à obtenir & 3.4 & 0.0 & 0.0 \\
\hline Difficile à utiliser & 0.9 & 1.3 & 0.0 \\
\hline Sexuellement inactive & 5.0 & 3.3 & 0.0 \\
\hline Fataliste & 0.8 & 1.3 & 0.0 \\
\hline Autre & 11.4 & 17.7 & 11.5 \\
\hline Total & 100.0 & 100.0 & 100.0 \\
\hline $\begin{array}{l}\text { Nombre de femmes ayant } \\
\text { arrêté l'utilisation de la méthode }\end{array}$ & 189 & 44 & 56 \\
\hline
\end{tabular}

Source: Base de données de l'Enquête Démographique et de Santé au Botswana de 1988.

Tableau 3 : Régression' du risque mensuel d'arrêter l'utilisation de la contraception sur un certain nombre de caractéristiques socio-économiques et culturelles (Botswana, 1988)

\begin{tabular}{|c|c|c|c|}
\hline $\begin{array}{l}\text { Caractéristiques socio- } \\
\text { économiques et culturelles } \\
\text { des femmes }\end{array}$ & $\begin{array}{l}\text { Coefficients } \\
\text { Estimés }\end{array}$ & $\begin{array}{l}\text { Ecarts } \\
\text { types }\end{array}$ & $\begin{array}{l}\text { Risques } \\
\text { relatifs }^{2}\end{array}$ \\
\hline Education & Référence & & \\
\hline Sans & -0.322 & 0.209 & ---- \\
\hline Primaire & -0.732 & 0.255 & 0.48 \\
\hline Secondaire ou plus & & & \\
\hline Religion & référence & & \\
\hline Chrétienne/protestante & 0.553 & 0.200 & $\mathrm{I} .74$ \\
\hline
\end{tabular}




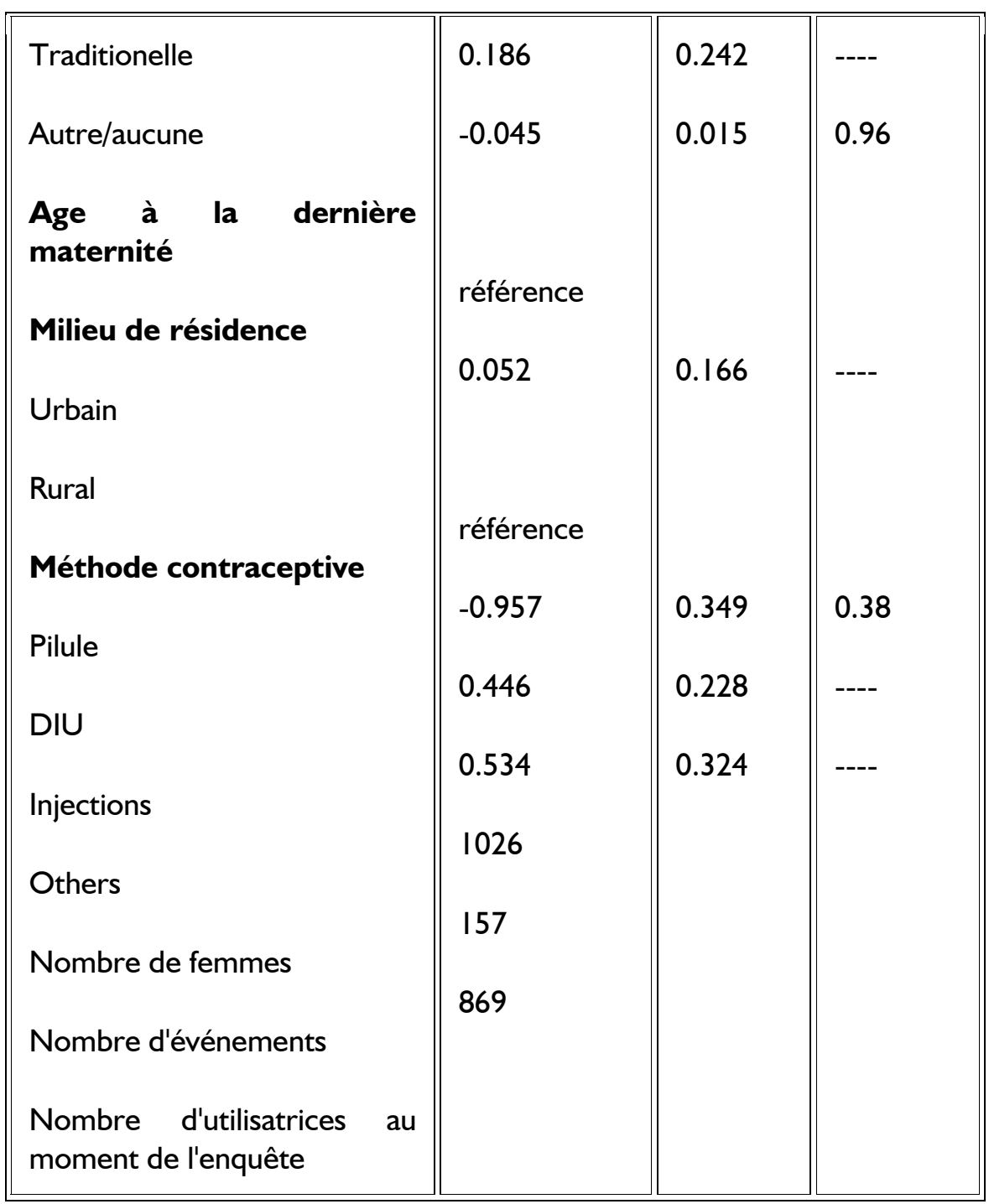

' La méthode de régression utilisée ici est celle proposée par Cox (1972) et connue sous le nom de model à risques proportionels.

${ }^{2}$ Le risque est exprimé ici relativement à la catégorie de référence. II est égal à l'exponentiel du coefficient estimé en colonne 2 du même tableau. Le risque relatif n'a pas été calculé lorsque le coefficient estimé n'est pas statistiquement différent de zéro au seuil de $5 \%$ ( c'est-à-dire

généralement lorsque le double de l'écart type est supérieur au coefficient estimé): dans ce cas il est sous-entendu que le risque relatif est égal à l'unité.

\section{BIBLIOGRAPHIE}

- Bruce J. and A. Jain: Improving the Quality of Care through Operations Research, in
Seidman M. and M.C. Horn: Operations Research: Helping Family Planning Programs Work Better, New York: WileyLiss, I99I, 259-282. 
- Bruce J.: Fundamental Elements of Quality of Care: A Simple Framework, Studies in Family Planning, 1990, 2(2):6I-9I.

- Bulatao, R.A.: Effective Family Planning Programs, Washington: the World Bank, 1993.

- Caldwell J.C. and P. Caldwell: Is the Asian Family Planning Program Model Suited to Africa?, Studies in Family Planning, 1988, 19(I): 19-28.Cox D.R.: Regression Models and Life Tables, Journal of the Royal Statistical Society, Serie B 34: 1972, I84-202.

- Dackam Ngatchou R., Mfoulou R., et SalaDiakanda M.: Population et santé familiale en Afrique Centrale, London: Fédération internationale pour la planification familiale, 1990.

- Foreit J.R.: Reaching More Users: More Methods, More Outlets, More Promotion, in Seidman M. and M.C. Horn: Operations Research: Helping Family Planning Programs Work Better, New York: Wiley-Liss, I99I, 2I5-23I.

- Grady W.R. and J.O.G. Billy: Effects of Demographic Factors on the Use and Effectiveness of New Contraceptive Technologies, in Segal S.J., A.O. Tsui and S.M. Rogers(eds): Demographic and Programmatic Consequences of Contraceptive Innovations, New York: Plenum Press, 1989, 73-104.

- Grady W.R., M.D. Hayward and F.A. Florey: Contraceptive Discontinuation among Married Women in the United States, Studies in Family Planning, 1988, 19(4): 227-235.

- Hammerslough C.R.: Characteristics of Women Who Stop Using Contraceptives, Family Planning Perspectives, 1984, I6(I): I4-I7.

- International Planned Parenthood Federation: Male Involvement in Family Planning, London: IPPF, 1984.

- Jain A.: Fertility Reduction and the Quality of Family Planning Services, Studies in Family Planning, 1989, 20(I): I-I6.

- Kraeger P.: Family Planning Drop-Outs Reconsidered: A Critical Review of Research and Research Findings, London: IPPF, 1977.
- Larson, K.: Botswana: Family Planning Myths and Beliefs, Unpublished mimeo, 1983.

- Lee N.C., G.L. Rubin and R. Boruck: The Intrauterine Device and Pelvic Inflammatory Diseases Revisited: New Results from the Women's Health Study, Obstetrics and Gyneacology, 1988, 72(I): I-6.

- Lesetedi L.T., G.D. Mompati, P. Khulumani, G.N. Lesetedi and N. Rutenberg: Botswana Family Health Survey II 1988, Columbia, Maryland: IRD/Macro Systems, Inc., I 989, 2 I-23.

- Mauldin W.P.: Experience with Contraceptive Methods in Developed Countries, The Population Council, Working Papers, I978, (30).

- Molnos A.: Attitudes Toward Family Planning in East Africa, München: Weltforum Verlag., 1968.

- Piotrow P.T. and R.C. Meyer: Promoting Family Planning: Findings from Operations Research and Program Research, in Seidman M. and M.C. Horn: Operations Research: Helping Family Planning Programs Work Better, New York: Wiley-Liss, I99I, 97-I42.

- Schirm A.L., J. Trussel, J. Menken and W.R. Grady: Contraceptive Failure in the United States: the Impact of Social, Economic and Demographic Factors, Family Planning Perspectives, 1982, I4(2): 68-75.

- Segal S.J.: Contraceptive Innovations: Needs and Opportunities,in Segal S.J., A.O. Tsui and S.M. Rogers(eds): Demographic and Programmatic Consequences of Contraceptive Innovations, New York: Plenum Press, 1989, 3-32.

- van de Walle E. \& F. van de Walle: Breastfeeding and Popular Aetiology in the Sahel, Health Transition Review, 199I, I(I): 69-8I.

- Williamson N.E., S. Thapa, and S. Balogh: Introducing New Methods, Popularizing Available Methods and Encouraging Correct Use and Continuation, in Seidman M. and M.C. Horn: Operations Research: Helping Family Planning Programs Work Better, New York: WileyLiss, 199I, 233-257. 


\section{REMERCIEMENTS}

L'auteur tient à remercier Etienne van de Walle et l'évaluateur anonyme du présent journal pour leurs suggestions et commentaires. Une version antérieure de cet article a été présentée à la conférence de I'UEPA sur "La Reproduction et la Santé Familiale en Afrique", Abidjan: 8-13/1 I/93. 